\title{
Impact of supernova feedback on the Tully-Fisher relation
}

\author{
M. E. De Rossi ${ }^{1,2}$, P. B. Tissera ${ }^{1,2}$, and S. E. Pedrosa ${ }^{1,2}$ \\ ${ }^{1}$ Consejo Nacional de Investigaciones Científicas y Técnicas, CONICET, Argentina \\ e-mail: derossi@iafe.uba.ar \\ 2 Instituto de Astronomía y Física del Espacio, Casilla de Correos 67, Suc. 28, 1428 Buenos Aires, Argentina \\ e-mail: [patricia; supe]@iafe.uba.ar \\ Received 13 January 2010 / Accepted 27 May 2010
}

\section{ABSTRACT}

\begin{abstract}
Context. Recent observational results have found a bend in the Tully-Fisher relation in such a way that low-mass systems lie below the linear relation described by more massive galaxies.

Aims. We intend to investigate the origin of the observed features in the stellar and baryonic Tully-Fisher relations and analyse the role played by galactic outflows on their determination.

Methods. Cosmological hydrodynamical simulations which include supernova feedback were performed in order to follow the dynamical evolution of galaxies.

Results. We found that supernova feedback is a fundamental process for reproducing the observed trends in the stellar Tully-Fisher relation. Simulated slowly rotating systems tend to have lower stellar masses than those predicted by the linear fit to the massive end of the relation, consistently with observations. This feature is not present if supernova feedback is turned off. In the case of the baryonic Tully-Fisher relation, we also detect a weaker tendency for smaller systems to lie below the linear relation described by larger ones. This behaviour arises as a result of the more efficient action of supernovae in the regulation of the star formation process and in the triggering of powerful galactic outflows in shallower potential wells, which may heat up and/or expel part of the gas reservoir.
\end{abstract}

Key words. galaxy: formation - galaxy: evolution - galaxy: structure

\section{Introduction}

The origin of the Tully-Fisher relation (Tully \& Fisher 1977) has been analysed by numerous observational and theoretical works since it provides important constraints on galaxy formation models (e.g. Avila-Reese et al. 1998; Mo et al. 1998; Avila-Reese et al. 2008). Originally defined as the relation between the luminosity and the rotation velocity for spiral galaxies, it is now accepted that this is a proxy for the more fundamental relation between stellar mass and rotation velocity (e.g. Conselice et al. 2005; Flores et al. 2006; Cresci et al. 2009) or even between baryonic mass and rotation velocity (e.g. Bell \& de Jong 2001; Verheijen 2001; Gurovich et al. 2004; McGaugh 2005; Puech et al. 2010) of the form $M \propto V^{\alpha}$. Observational evidence suggests there is a break in the stellar Tully-Fisher relation (sTFR) at a rotation velocity of $\sim 90 \mathrm{~km} \mathrm{~s}^{-1}$ (McGaugh et al. 2000; Amorín et al. 2009). Even more, recently McGaugh et al. (2010) have also reported a break in the baryonic Tully-Fisher relation (bTFR) but at a lower velocity.

Assuming that galaxies formed in virialized haloes and that the percentage of gas transformed into stars is independent of halo mass, a relation between stellar mass and circular velocity with a slope of $\alpha \sim 3$ is directly obtained (White \& Frenk 1991; Mo et al. 1998). This theoretical prediction is reproduced in cosmological simulations when ineffective mechanisms to regulate the gas cooling and star formation activity are introduced (Tissera et al. 1997; Steinmetz \& Navarro 1999). Therefore, changes in the slope of this relation to match observed reported values, which are on average close to $\alpha \sim 4$, might require the action of other physical processes. Theoretical findings suggest that the triggering of powerful mass-loaded galactic winds by massive supernova (SN) explosions would have an efficiency that depends on the virial halo so that stronger effects are expected in low-mass systems (Larson 1974). In fact, Dekel \& Silk (1986) estimated analytically that haloes with circular velocity lower than $\sim 100 \mathrm{~km} \mathrm{~s}^{-1}$ would be strongly affected by massive SN events. Nonetheless, the role of galactic outflows on the origin of the relations between the stellar and dynamical properties of dwarf galaxies still constitutes an open problem (Mac Low \& Ferrara 1999; Tassis et al. 2008). In a cosmological framework, only recently has it been possible to start simulating the triggering of galactic outflows on more physical basis (e.g. Scannapieco et al. 2006; Stinson et al. 2007; Governato et al. 2010). In fact, cosmological simulations that include this process obtained sTFRs in more qualitative agreement with observations (Governato et al. 2007; Croft et al. 2009). There is evidence that the implementation of a suitable feedback model could account for a bend in the TFR as suggested by semi-analytical works (e.g. Kang et al. 2005; Nagashima et al. 2005; Dutton \& van den Bosch 2009; Guo et al. 2010) and some hydrodynamical simulations (e.g. Tassis et al. 2008). However, a detailed analysis of the effects of SN feedback on the TFR using a physically motivated model was still missing.

In this paper, we present results on the analysis of the sTFR and bTFR by using a SN feedback model especially suitable to studying the formation of galaxies in cosmological scenarios (Scannapieco et al. 2005, 2006), since it does not include any scale-dependent parameter and it is capable to produce the expected anti-correlation between the strength of the galactic outflows and the potential well of the haloes, as shown by Scannapieco et al. (2006).

This paper is organized as follows. Section 2 describes the numerical experiments. Section 3 outlines the results. In Sect. 4 we discuss about our main findings, and in Sect. 5 we present the conclusions of this work. 
Table 1. Cosmological hydrodynamical simulations studied in this paper.

\begin{tabular}{lcccc}
\hline \hline Name & F/NF & $N_{\mathrm{p}}$ & $\begin{array}{c}M_{\text {dark }} \\
{\left[10^{6} M_{\odot} h^{-1}\right]}\end{array}$ & $\begin{array}{c}M_{\text {gas }} \\
{\left[10^{6} M_{\odot} h^{-1}\right]}\end{array}$ \\
\hline S160 & F & $2 \times 160^{3}$ & 17.6 & 2.71 \\
S230 & F & $2 \times 230^{3}$ & 5.93 & 0.91 \\
S230NF & NF & $2 \times 230^{3}$ & 5.93 & 0.91 \\
S320 & F & $2 \times 320^{3}$ & 2.20 & 0.34 \\
\hline
\end{tabular}

Notes. Column 2: Model with/without SN feedback (F/NF). Column 3: Initial number of particles in the simulation. Column 4: Mass of dark matter particles. Column 5: Initial mass of gas particles.

\section{Numerical experiments}

We performed cosmological simulations of a typical field region of the Universe consistent with the concordance model. We adopt the following cosmological parameters: $\Omega_{\Lambda}=0.7$, $\Omega_{\mathrm{m}}=0.3, \Omega_{\mathrm{b}}=0.04$, a normalization of the power spectrum of $\sigma_{8}=0.9$ and $H_{0}=100 h \mathrm{~km} \mathrm{~s}^{-1} \mathrm{Mpc}^{-1}$, with $h=0.7$. Two of the simulations were run with $2 \times 230^{3}$ (S230) particles, while a third one and a fourth one used $2 \times 320^{3}$ (S320) and $2 \times 160^{3}$ (S160) to check numerical effects. In all cases, the simulated volumes correspond to a cubic box of a comoving $10 \mathrm{Mpc}^{-1}$ side length. The particle masses for the dark and initial gas components are given in Table 1. Simulation S320 could only reach $z \approx 2$ due to high computational costs. By analysing the sTFR and bTFR in S230, S320, and S160, we check our results for numerical effects.

The simulations were performed by using a version of GADGET-3, an update of GADGET-2 optimized for massively parallel simulations of highly inhomogeneous systems (Springel \& Hernquist 2003; Springel 2005). This version of GADGET-3 includes the multiphase model for the interstellar medium (ISM) and the SN feedback scheme of Scannapieco et al. (2005, 2006). We ran the same initial condition with (S230) and without SN feedback (S230NF) in order to quantify the effects of $\mathrm{SN}$-induced galactic winds.

The SN feedback scheme adopted in this work considers both type II and type Ia SNe for the chemical and energy production. We adopt $0.7 \times 10^{51}$ erg per SN event. The chemical evolution model used in this code is the one developed by Mosconi et al. (2001) and, later on, adapted by Scannapieco et al. (2005) for GADGET-2. This model assumes the instantaneous recycling approximation (IRA) for SNII ${ }^{1}$. Lifetimes for the progenitors of SNIa are randomly selected in the range $\left[10^{8}, 10^{9}\right] \mathrm{yr}$. The chemical yields for SNII are given by Woosley \& Weaver (1995), while those of SNIa correspond to the W7 model of Thielemann et al. (1993).

The SN feedback model is grafted onto a multiphase model especially designed to improve the description of the ISM, allowing the coexistence of diffuse and dense gas phases. Within this new framework, the injection of energy and heavy elements into different components of the ISM can be more realistically treated, leading to a more effective production of galactic winds. The ejected energy is distributed into and managed differently by the so-called cold (temperature $T<T_{\mathrm{c}}$ where $T_{\mathrm{c}}=8 \times 10^{4} \mathrm{~K}$ and density $\rho>0.1 \rho_{\mathrm{c}}$ where $\rho_{\mathrm{c}}$ is $7 \times 10^{-26} \mathrm{~g} \mathrm{~cm}^{-3}$ ) and hot

\footnotetext{
1 Scannapieco et al. (2005) also implemented a version that relax the IRA, assuming the age-mass-metallicity fitting polynomials estimated by Raiteri et al. (1996). However, no significant changes were found in the results compared to the IRA.
}

(otherwise) ISM components. The hot phase thermalizes instantaneously the energy, while the cold phase builds up a reservoir until it accumulates enough energy to raise the entropy of the gas particle to match the value of its own surrounding hot environment. The properties of the hot and cold gas components of each gas particle are estimated locally without using any global properties of the systems. The percentage of SN energy distributed into the cold phase is given by the feedback parameter $\epsilon_{\mathrm{c}}$. The ejection and distribution of chemical elements is coupled to the energy procedure so that the percentage of metals pumped into the cold and hot phases are also given by $\epsilon_{\mathrm{c}}$ in these simulations. We adopt $\epsilon_{\mathrm{c}}=0.5$, which was found by Scannapieco et al. (2008) to best reproduce a disc galaxy in simulations of a Milky-Way type halo.

Virialized structures are selected from the general mass distribution by using a standard friends-of-friends technique. The substructures residing within a given virialized halo are then individualized with the SUBFIND algorithm of Springel et al. (2001) to build up galaxy catalogues at different redshifts. By definition, a substructure can correspond either to the central galaxy or a satellite system within a given halo. To characterize the simulated galaxies, we define the baryonic radius $\left(R_{\mathrm{bar}}\right)$ as the one that encloses $83 \%$ of the baryons associated to each substructure. In the case of S160, S230 and S230NF, for all the linear regressions, we only consider those simulated galaxies defined by more than $2 \times 10^{3}$ particles within $R_{\text {bar }}$, which is equivalent to have stellar masses greater than $10^{9} M_{\odot} h^{-1}$. In S230, Milky-Way type galaxies at $z=0$ are resolved with approximately $10^{5}$ total particles within $R_{\mathrm{bar}}$. A similar procedure was carried out for the high-resolution simulation S320, where Milky-Way type systems are already resolved with more than $10^{5}$ particles within $R_{\text {bar }}$ at $z=2.0$. In S320, the smallest simulated galaxies we considered for calculations have $10^{4}$ particles within $R_{\text {bar }}$.

The properties of the simulated galaxies, such as stellar mass, baryonic mass and rotation curves are estimated within $R_{\text {bar }}$. We define disc systems as those with more than $75 \%$ of their gas component on a rotationally supported disc structure by using the condition $\sigma / V<1$ to select them (where $\sigma$ and $V$ are the velocity dispersion and the tangential velocity component, respectively). We acknowledge that, at $z=0$, most of our galaxies contains large stellar bulges and thick stellar discs, which are more consistent with early type spirals. Nevertheless, the gaseous disc components are very well-defined and trace the potential well of their host haloes remarkably well as can be appreciated in Fig. 1. Rotation curves are estimated by using the tangential velocity of gas particles on the plane perpendicular to their total angular momentum. For these systems, tangential velocity constitutes a good representation of the potential well of the system. For the sake of simplicity, we use $V_{\text {cir }}^{2}=G M(r<R) / R($ where $M(r<R)$ is the enclosed total mass within $R$ ) estimated at $R_{\mathrm{bar}}$ as an indicator of the gravitational mass for systems in both S230 and S230NF.

\section{Results}

We calculated the STFR for our simulated galaxies by fitting a relation of the form $\log M_{*}=\alpha \log \left(V_{\text {cir }} / 100 \mathrm{~km} \mathrm{~s}^{-1}\right)+Y_{100}$. In the case of the local sTFR in S230, we found a slope of $3.68 \pm 0.09$ and $Y_{100}=9.42 \pm 0.26$, in general agreement with observations (McGaugh et al. 2000; Bell \& de Jong 2001). As can be seen from Fig. 2 (upper left panel), the residuals of the linear fit to the relation (small box) depart systematically from zero at around $100 \mathrm{~km} \mathrm{~s}^{-1}$, so that systems with lower circular velocities tend 
M. E. De Rossi et al.: SN feedback and the Tully-Fisher relation
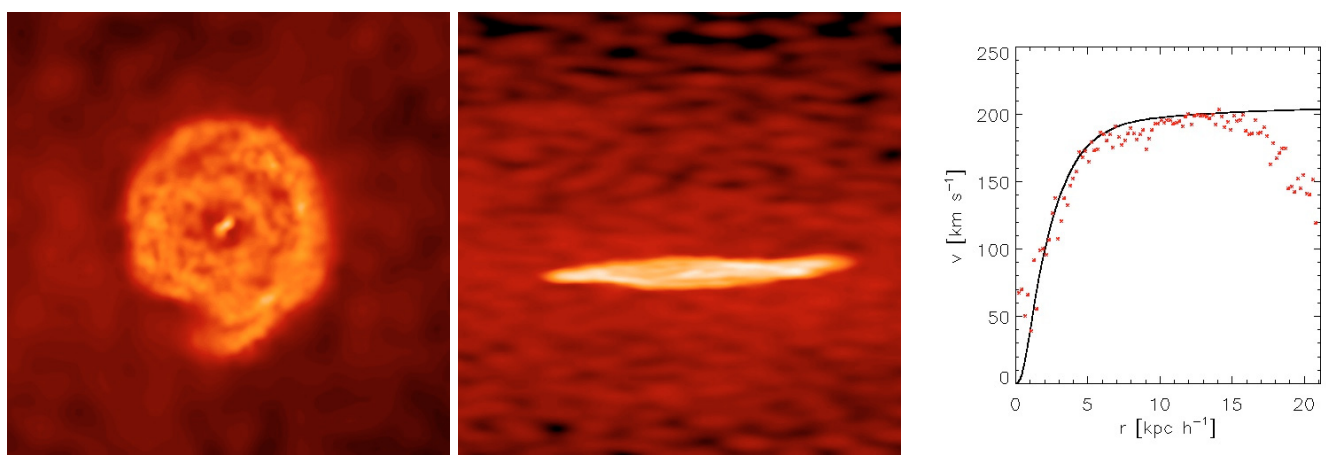

Fig. 1. Projected gaseous mass distributions for a typical disc galaxy in a face-on view (left panel) and an edge-on one (middle panel) and the corresponding $V_{\text {rot }}$ (dots) and $V_{\text {circ }}$ (continuous line) for this system (right panel) in $\mathrm{S} 230$. For this galaxy, $R_{\mathrm{bar}}$ is $\approx 13.7 \mathrm{kpc} \mathrm{h}^{-1}$.
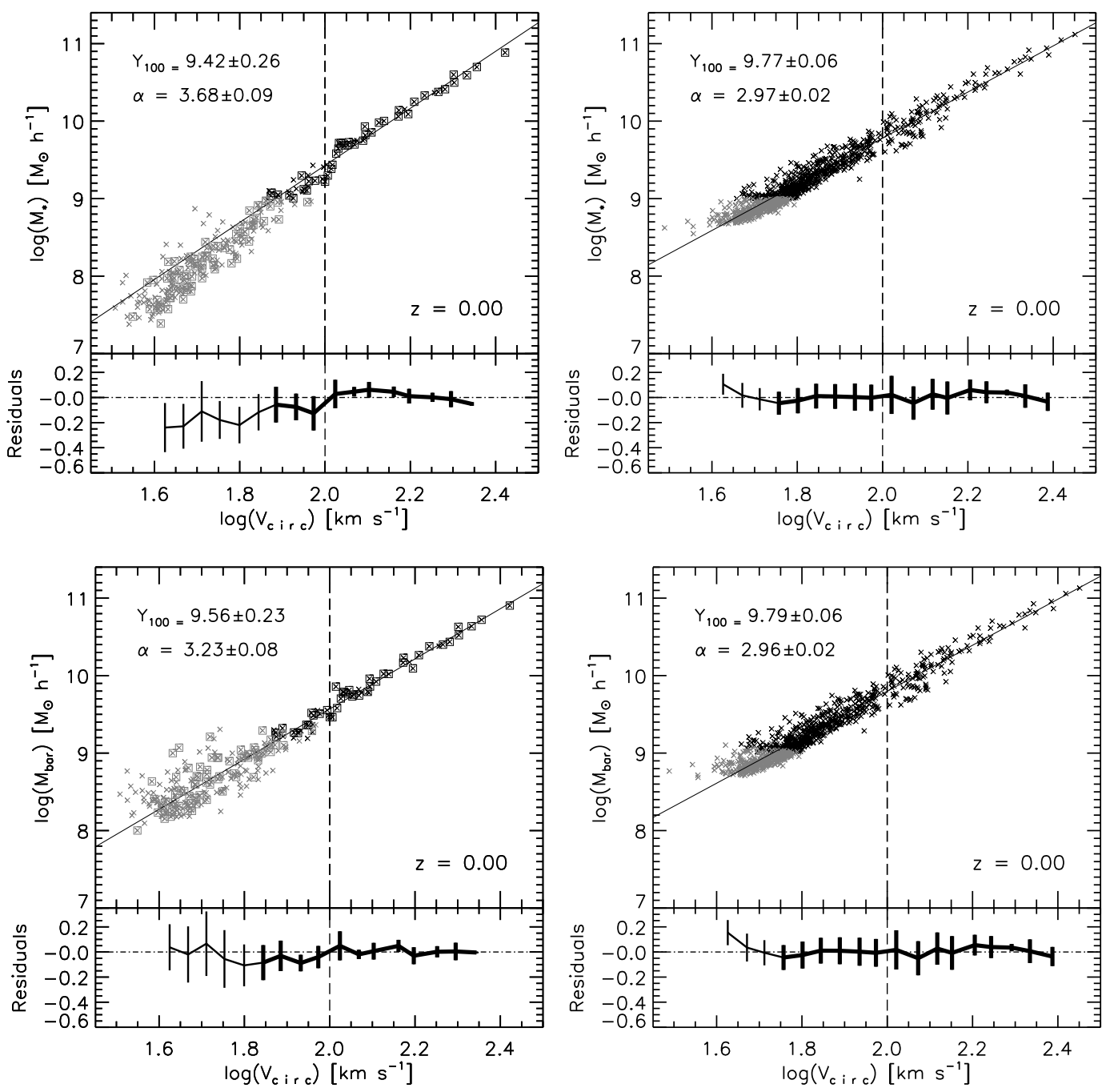

Fig. 2. The sTFR (upper panels) and bTFR (lower panels) in the S230 (left) and S230NF (right) runs, at $z=0$. Grey symbols depict low-mass systems $\left(M_{*}<10^{9} M_{\odot} h^{-1}\right)$, while massive ones $\left(M_{*}>10^{9} M_{\odot} h^{-1}\right)$ are coloured in black. Systems in the S230 run, with well-defined gaseous discs, are enclosed with squares. The black line corresponds to the linear fit. The values of the slope $(\alpha)$ and $Y_{100}$ are indicated in the figure. The vertical lines indicates the velocity where the residuals (small boxes) of the sTFR in S230 depart systematically from zero. The thicker lines denote the range of velocities associated with stellar masses over $10^{9} M_{\odot} h^{-1}$.

to have lower stellar masses than the predictions obtained from the fitting. Interestingly, this trend is consistent with the observations reported by McGaugh et al. (2000) and Amorín et al. (2009) and from theoretical expectations (Larson 1974; Dekel \& Silk 1986). To analyse to what extent SN feedback might be responsible for this behaviour, we compared these findings with the ones obtained for S230NF as shown in the upper right panel of Fig. 2. We can appreciate that $\mathrm{SN}$ feedback seems to be crucial for reproducing the observed features in the sTFR, since when this mechanism is turned off, the simulated sTFR exhibits 
Table 2. Results of the linear fits to the high-mass end $\left(M_{*}>\right.$ $10^{9} M_{\odot} h^{-1}$ ) of the sTFR for S230 at $z=0$.

\begin{tabular}{lcc}
\hline \hline$V_{\mathrm{i}}$ & $Y_{100}$ & $\alpha$ \\
\hline$V_{\text {circ }}$ at $0.5 R_{\text {bar }}$ & $9.51 \pm 0.26$ & $3.62 \pm 0.09$ \\
$V_{\text {circ }}$ at $R_{\text {bar }}$ & $9.42 \pm 0.26$ & $3.68 \pm 0.09$ \\
$V_{\text {circ }}$ at $1.5 R_{\text {bar }}$ & $9.43 \pm 0.25$ & $3.59 \pm 0.08$ \\
$V_{\max }$ & $9.41 \pm 0.25$ & $3.67 \pm 0.08$ \\
\hline
\end{tabular}

Notes. Column 1: Kinematic indicator used for the calculations. Column 2: Stellar mass corresponding to a velocity of $100 \mathrm{~km} \mathrm{~s}^{-1}$ according to the fitting. Column 3: Slope derived from the linear regression.

a linear behaviour with a slope of $\sim 3$, in agreement with theoretical predictions. We can also see that the wind-free run predicts higher stellar masses for systems at a given circular velocity, since it cannot regulate the transformation of gas into stars, which is too efficient in hydrodynamical simulations.

We also studied the bTFR in S230 and S230NF (Fig. 2, lower panels). The bTFR is obtained by adding all baryons within $R_{\mathrm{bar}}$, regardless of its physical state. We can see that both models (with and without SN feedback) predict a linear trend for the bTFR, at least, for the range of velocities covered by these simulations. At $z=0$, the simulated bTFR in S230 has a slope of $3.23 \pm 0.08$ and $Y_{100}=9.56 \pm 0.23$. These values are generally in good agreement with observational results reported for late and early type galaxies (De Rijcke et al. 2007; Gurovich et al. 2010). However, we warn that the comparison with observations is tricky since we sum up the total amount of gas, while observers are limited by the instrumental techniques that only give access to gas mass with certain physical properties. For S230NF, the bTFR has a higher value of $Y_{100}$ because these galaxies have been able to retain their baryons in the central regions since no SN-driven outflows could be triggered. Also, S230NF yields similar fittings to the sTFR and bTFR, indicating that stars dominate the baryonic phase at this redshift.

As already mentioned, the previous analysis was done by using the value of $V_{\text {circ }}$ at $R_{\text {bar }}$ as a kinematical indicator. To analyse the dependance of our results on the particular choice of the radius at which we measure $V_{\text {circ }}$, we also compared the relations obtained by using $V_{\text {circ }}$ evaluated at $0.5 R_{\text {bar }}$ and $1.5 R_{\text {bar }}$. We also estimated the maximum value of the rotation curve $V_{\max }$ as can be seen from Fig. 3. The simulated sTFR and bTFR are not significantly affected by variations in the radius at which $V_{\text {circ }}$ is estimated. The largest changes with respect to our previous results (Fig. 2) are obtained when using $V_{\text {circ }}$ at $0.5 R_{\text {bar }}$ as the velocity estimator. Nevertheless, all changes remain within a $\sigma$. It is also worth noting that, in these simulations, $V_{\text {circ }}$ at $R_{\text {bar }}$ constitutes a good proxy for the velocity $V_{\max }$, which is commonly employed in many observational works. Tables 2 and 3 summarize the parameters corresponding to the linear fits to the highmass end $\left(M_{*}>10^{9} M_{\odot} h^{-1}\right)$ of the TFRs shown in Fig. 3. We can appreciate that, in all cases, the values of $\alpha$ and $Y_{100}$ agree within a $\sigma$. Regarding the characteristic velocity where the sTFR bends, our findings suggest that it does not depend on the particular kinematical estimators tested in this paper (Fig. 3, small boxes). In light of these results, we hereafter use $V_{\text {circ }}$ at $R_{\text {bar }}$ as the kinematical indicator for our calculations.

By comparing the sTFR and bTFR in S230, it is clear that adding the gas mass in the calculations contributes to restoring the linearity of the TFR over a wider velocity range. These findings suggest that, at $z \sim 0$, the bend of the local sTFR might be partially caused by a decrease in the star formation rate in
Table 3. Results of the linear fits to the high-mass end $\left(M_{*}>\right.$ $10^{9} M_{\odot} h^{-1}$ ) of the bTFR for S230 at $z=0$.

\begin{tabular}{lcc}
\hline \hline$V_{\mathrm{i}}$ & $Y_{100}$ & $\alpha$ \\
\hline$V_{\text {circ }}$ at $0.5 R_{\text {bar }}$ & $9.64 \pm 0.20$ & $3.19 \pm 0.07$ \\
$V_{\text {circ }}$ at $R_{\text {bar }}$ & $9.56 \pm 0.23$ & $3.23 \pm 0.08$ \\
$V_{\text {circ }}$ at $1.5 R_{\text {bar }}$ & $9.57 \pm 0.25$ & $3.14 \pm 0.08$ \\
$V_{\max }$ & $9.55 \pm 0.24$ & $3.22 \pm 0.08$ \\
\hline
\end{tabular}

Notes. Column 1: Kinematic indicator used for the calculations. Column 2: Baryonic mass corresponding to a velocity of $100 \mathrm{~km} \mathrm{~s}^{-1}$ according to the fitting. Column 3: Slope derived from the linear regression.

smaller systems as a consequence of the gas heating by SNe. To quantify to what extent $\mathrm{SN}$ feedback can generate a decrease in the star formation activity of simulated galaxies, we calculated the star formation efficiency (eSFR) defined as the ratio of the star formation rate of a given galaxy to the total amount of gas contained within it. In Fig. 4, we compare the mean eSFR as a function of $V_{\text {circ }}$ for galaxies in $\mathrm{S} 230$ and $\mathrm{S} 230 \mathrm{NF}$ at $z=0$. As expected, the model that includes $\mathrm{SN}$ feedback predicts smaller eSFRs at a given $V_{\text {circ }}$, with slowly rotating systems exhibiting the most important variations, on average. These systems still have an important percentage of gas but it is in the form of a diffuse warm environment that does not fulfil the conditions for forming stars.

To analyse the evolution of both the sTFR and bTFR as a function of redshift, we performed linear fits to the fast rotators using the characteristic velocity $V_{\text {circ }}=100 \mathrm{~km} \mathrm{~s}^{-1}$ as a reference value at each analysed redshift. As can be seen in Fig. 5, the residuals of the sTFR show a systematic departure from zero already from $z \approx 3$, which occurs at approximately a similar characteristic velocity of $\sim 100 \mathrm{~km} \mathrm{~s}^{-1}$. For this $\mathrm{SN}$ model, we detect an evolution in the $Y_{100}$ of the sTFR of $\approx 0.44$ dex since $z \approx 3$, while the slope of the fast-rotating systems remains almost constant. In the case of the bTFR, $Y_{100}$ evolves by around $\sim 0.30$ dex between $z=3$ and $z=0$. In particular, as can be appreciated from Fig. 5, the simulated bTFR retains the linear relation over a wider velocity range. At $z=0$, there is no clear signal for a change in the slope within the numerical dispersion. This finding is not in conflict with the recent observational results of McGaugh et al. (2010), who report a break in the bTFR at $\sim 20 \mathrm{~km} \mathrm{~s}^{-1}$, because our simulations do not numerically resolve such low velocity systems. While better statistics and higher numerical resolution are needed to test the behaviour of the bTFR for low-velocity systems, more detailed observations are also required to robustly probe the existence of the bend in the bTFR. With regard to the S230NF, both the sTFR and the bTFR exhibit a linear trend from $z=3$ with no significant changes in the slope but with an evolution of $\approx 0.55$ in $Y_{100}$, which is consistent with the higher eSFR measured in the galaxies in this run. Both TFRs show larger dispersion for low-velocity rotators, which are also more gas rich than systems at the high-velocity end. At a given velocity, slow rotators can have different baryonic and stellar masses, indicating their different evolutionary paths. This behaviour can be also seen in S230NF albeit weaker, so the dispersion has at least two causes: the different evolutionary paths at a given circular velocity that regulate the transformation of gas into stars and the action of SN feedback.

To assess the effects of numerical resolution on the determination of characteristic velocity $V_{\mathrm{c}} \sim 100 \mathrm{~km} \mathrm{~s}^{-1}$, we performed linear fits to the massive end $\left(M_{*}>10^{9} M_{\odot} h^{-1}\right)$ of the sTFR and bTFR in S320, S230, and S160 at $z=2.0$. In Fig. 6, we compare 

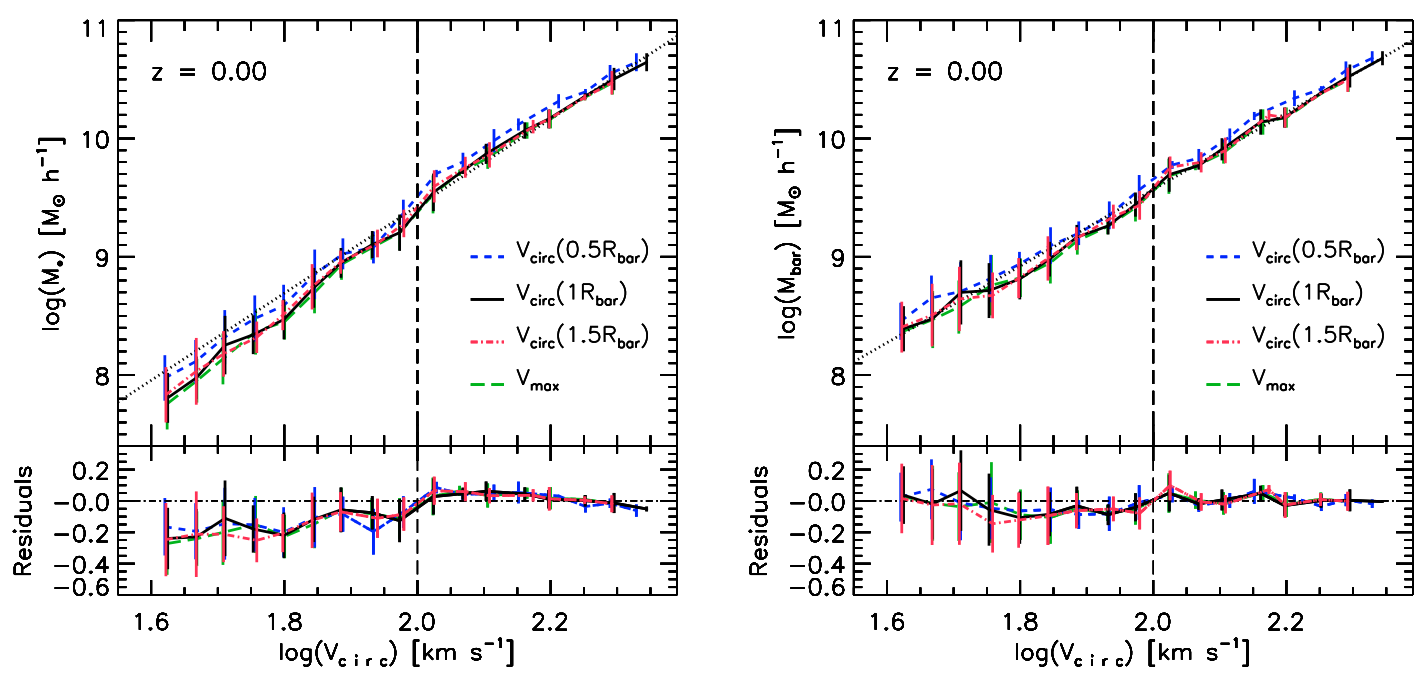

Fig. 3. Mean sTFR (left panel), bTFR (right panel), and the corresponding standard deviations for S230 at $z=0$. Results obtained by using different kinematic indicators are compared: $V_{\text {circ }}$ at $0.5 R_{\mathrm{bar}}, R_{\mathrm{bar}}$ and $1.5 R_{\mathrm{bar}}$, and the maximum value of the rotation curve $V_{\mathrm{max}}$. The dotted black lines represent the fittings to the high-mass end $\left(M_{*}>10^{9} M_{\odot} h^{-1}\right)$ of the simulated TFRs when using $V_{\text {circ }}$ at $R_{\text {bar }}$ as kinematic indicator. The vertical line depicts the characteristic velocity where the STFR bends. The small boxes show the residuals of each TFR with respect to the linear fit to its high-mass end.

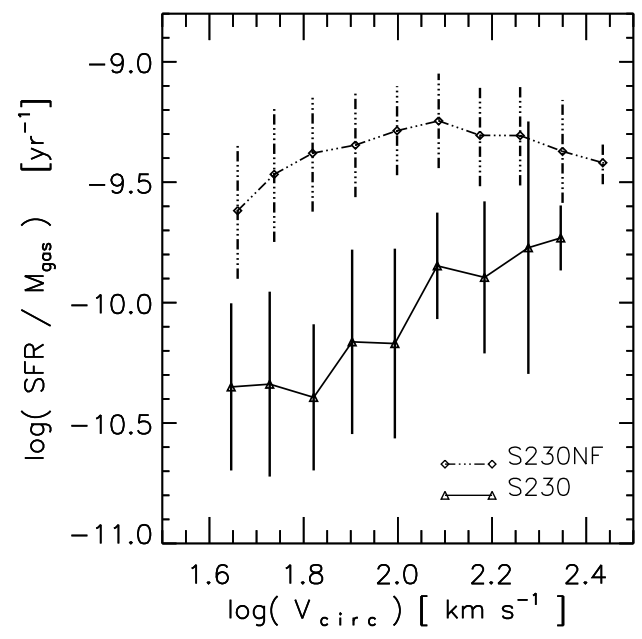

Fig. 4. Mean star formation efficiency (eSFR) as a function of $V_{\text {circ }}$ for galaxies in S230 (triangles) and in S230NF (diamonds) at $z=0$.

the mean values and standard deviations of the residuals associated to each simulation. We also show the results for S320. As can be seen, the trend for the sTFR is clearly present in all simulations, showing a change in the slope at a similar velocity. With respect to the bTFR, it also exhibits a bend in the residuals at around $\sim 100 \mathrm{~km} \mathrm{~s}^{-1}$ at $z \sim 2$, but the trend is weaker than for the sTFR. The three simulations agree on the existence of the departure from linearity at approximately the same velocity. In the three runs, we also find larger dispersions for low-velocity rotators. The agreement between the three different resolutionlevel simulations suggests that these results are robust against numerical artefacts.

Finally, since the SN feedback model uses $T_{\mathrm{c}}=8 \times 10^{4} \mathrm{~K}$ (equivalent to $\log V_{\text {vir }} \approx 1.95$, where $V_{\text {vir }}$ is the virial velocity of the halo) as a parameter to separate cold and hot gas-phases surrounding stellar populations, we evaluated how far this selection can influence the determination of the characteristic velocity where the sTFR bends. To assess this issue, we performed an extra simulation (S230b) with the same initial conditions and SN and star formation parameters of $\mathrm{S} 230$, except for $T_{\mathrm{c}}$, which was lowered to $5 \times 10^{4} \mathrm{~K}$ (equivalent to $\log V_{\text {vir }} \approx 1.84$ ). Figure 6 shows that for both TFRs the residuals of these simulations are very similar. Particularly, both simulations predict a bend at $\sim 100 \mathrm{~km} \mathrm{~s}^{-1}$ independently of the adopted value of $T_{\mathrm{c}}$, indicating that this parameter has no significant effect of the determination of the bend.

\section{Discussion}

To further investigate our findings regarding the origin of the bend in the TFR and the role played by SN feedback, we studied the fraction $f_{i}=\Omega_{\mathrm{m}} M_{i} / \Omega_{\mathrm{b}} M_{\mathrm{vir}}$ for each simulated galaxy at $z=0$, where $M_{\mathrm{vir}}$ is the total mass within the virial radius and $i$ denotes the stellar or the baryonic component within $R_{\mathrm{bar}}$. The quantity $f_{*}\left(f_{\mathrm{b}}\right)$ therefore represents the ratio between the stellar (baryonic) mass in a given galaxy and the expected baryonic mass within the virial radius inferred from the universal baryonic fraction $\left(\Omega_{\mathrm{b}} / \Omega_{\mathrm{m}}\right)$ corresponding to the adopted cosmological parameters. As can be appreciated from Fig. $7, f_{*}$ is an increasing function of the circular velocity that, in its turn, is a measure of the potential well of the systems. The small systems tend to have $f_{*}<0.1$ at $z=0$, while $f_{*}$ reaches $\sim 0.3$ for more massive galaxies. These findings are consistent with the fact that $\mathrm{SN}$ outflows are more efficient to heat up and/or expel the gas content from star-forming regions in shallower potential wells, leading to a decrease in their stellar mass content and making them lie below the sTFR determined by massive galaxies. At $z=2$, the trends are similar to the local ones with faster rotators systems exhibiting stellar fractions of around 0.4 and most of the slow rotators not exceeding 0.2. Nonetheless, at a given circular velocity, the discrepancies between the simulated stellar components and the theoretical baryonic masses are more significant in the local Universe, showing the accumulated effects of $\mathrm{SN}$ feedback. We also note that high redshift galaxies show a higher slope for the relation between $f_{*}$ and $V_{\mathrm{c}}$ indicating the 

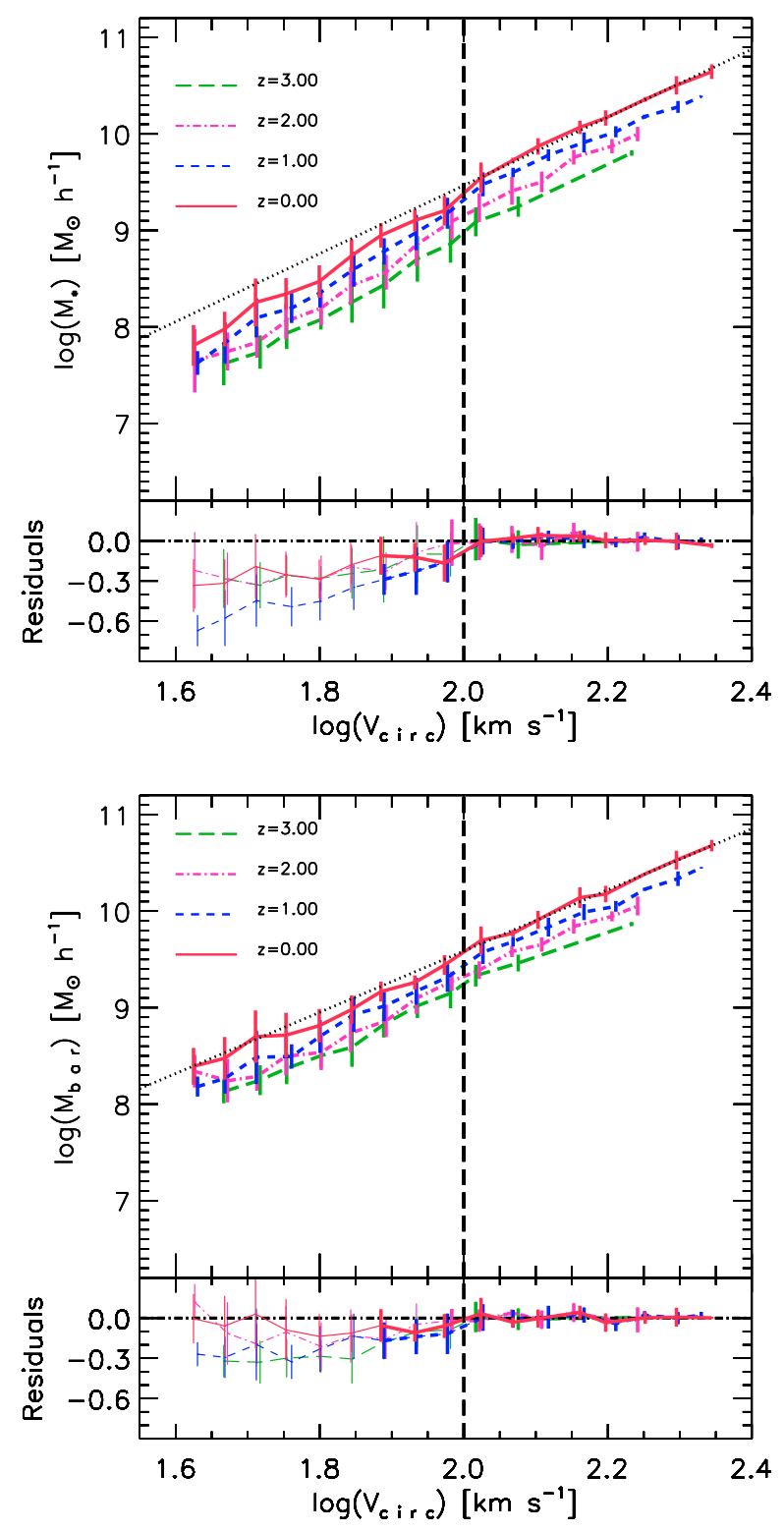

Fig. 5. The evolution of the mean sTFR (upper panel) and bTFR (lower panel) as a function of redshift for S230 for $z=3,2,1$, and 0 . The dotted black lines correspond to the fittings to the simulated TFRs for systems with $V_{\text {circ }}>100 \mathrm{~km} \mathrm{~s}^{-1}$ at $z=0$. The vertical line indicates this characteristic velocity. In the small boxes we included the residuals estimated with respect to the corresponding linear regressions calculated at each redshift. The thicker lines in the residuals indicate the velocities associated with stellar masses higher than $10^{9} M_{\odot} h^{-1}$.

more important action of $\mathrm{SN}$ winds on smaller systems at this epoch.

When the gas mass within $R_{\text {bar }}$ is incorporated into the calculations, we obtained the total baryonic fraction $f_{\mathrm{b}}$. At $z=0, f_{\mathrm{b}}$ shows a weaker dependence on circular velocities than $f_{*}$. More massive galaxies exhibit $f_{\mathrm{b}} \sim f_{*}$, indicating that these systems are gas poor. On the other hand, small galaxies exhibit baryonic fractions $f_{\mathrm{b}}$ that are more than half the value of $f_{*}$. These correlations explain the trend to recovering a single-slope relation for the local TFR over a broader velocity range when considering the baryonic content of galaxies instead of their stellar mass. However, that $f_{\mathrm{b}}<0.55$ within $R_{\mathrm{bar}}$ for our whole sample indicates that galactic outflows are important over the whole

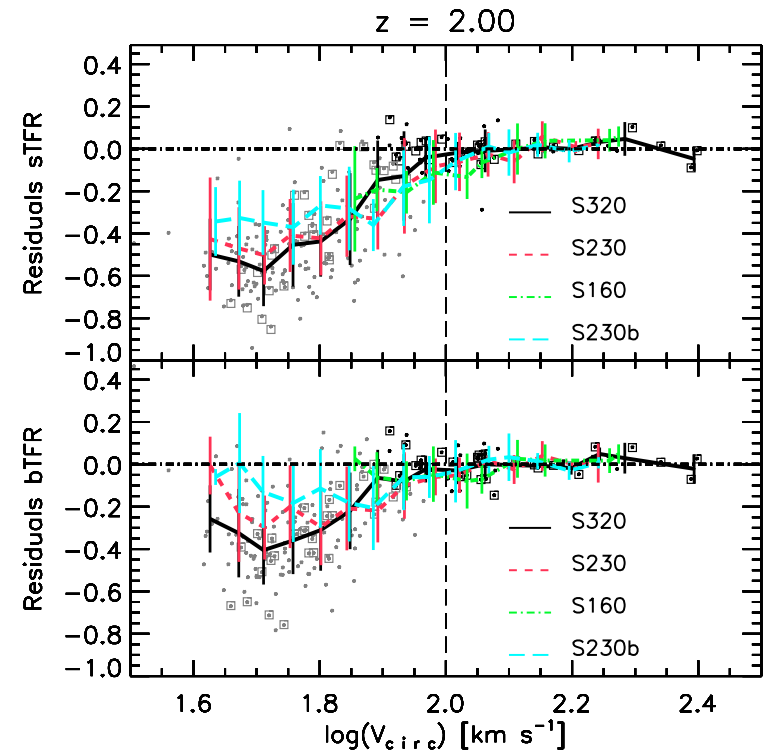

Fig. 6. Residuals corresponding to the linear fits to the massive end $\left(M_{*}>10^{9} M_{\odot} h^{-1}\right)$ of the sTFR (upper panel) and bTFR (lower panel) in S320 (symbols) at $z=2.0$. Grey symbols depict low-mass systems $\left(M_{*}<10^{9} M_{\odot} h^{-1}\right)$, while massive ones $\left(M_{*}>10^{9} M_{\odot} h^{-1}\right)$ are coloured in black. Systems with well-defined gaseous discs are enclosed with squares. The different lines depict the mean values and standard deviations associated to the residuals of the linear fits of S320, S230, S160, and $\mathrm{S} 230 \mathrm{~b}$.

simulated sample. The effects on the faster rotators are also indirect since they grow by the accretion of smaller substructures, which are strongly affected by SN feedback, as shown before. The $f_{*}$ and $f_{\mathrm{b}}$ predicted by simulations qualitatively agree with observations (e.g. McGaugh et al. 2010), albeit with a weaker dependence on $M_{*}$. At $z=2$, on the other hand, we obtained a stronger correlation between $f_{\mathrm{b}}$ and $V_{\text {circ }}$. Slow-rotating systems at this redshift show, on average, similar values of $f_{\mathrm{b}}$ to local ones. Conversely, fast rotators in the local Universe exhibit half of the baryonic fractions derived for systems of similar circular velocities at $z=2$. This behaviour is consistent with the stronger signal for a bend in the bTFR that we found at high redshifts and shows that $\mathrm{SN}$ feedback has acted on these systems by expelling part of the baryonic mass outside the very central regions from $z \sim 2$.

To estimate whether the expelled baryonic mass is within the virial radius or if $\mathrm{SN}$ outflows have been powerful enough to transport it into the intergalactic medium (IGM), we calculated the fraction $f_{\mathrm{b}}^{\mathrm{vir}}$ of the total baryonic mass within the virial radius of simulated galaxies relative to the one derived from the universal baryonic fraction $\left(\Omega_{\mathrm{b}} / \Omega_{\mathrm{m}}\right)$ in the adopted cosmology. The results can be appreciated in the right hand panels of Fig. 7. By comparing these findings with the ones obtained for $f_{\mathrm{b}}$, it is clear that a large amount of the missing baryons within $R_{\text {bar }}$ can be found in the surrounding halo. However, given that $f_{\mathrm{b}}^{\text {vir }}<1$, there is also a percentage of the gas blown away as a consequence of very efficient galactic winds. These effects are more evident at lower redshifts and for smaller systems.

We can also see that the correlation with virial mass is clearly defined at both redshifts though with high dispersion. At $z=2$, the percentage of absent baryons within $R_{\text {vir }}$ ranges from $20 \%$ to $70 \%$, with slow rotators having experienced the most significant losses, as expected. In the case of local galaxies, the percentage of lost baryons is within the range $30 \%$ and $80 \%$. 
M. E. De Rossi et al.: SN feedback and the Tully-Fisher relation
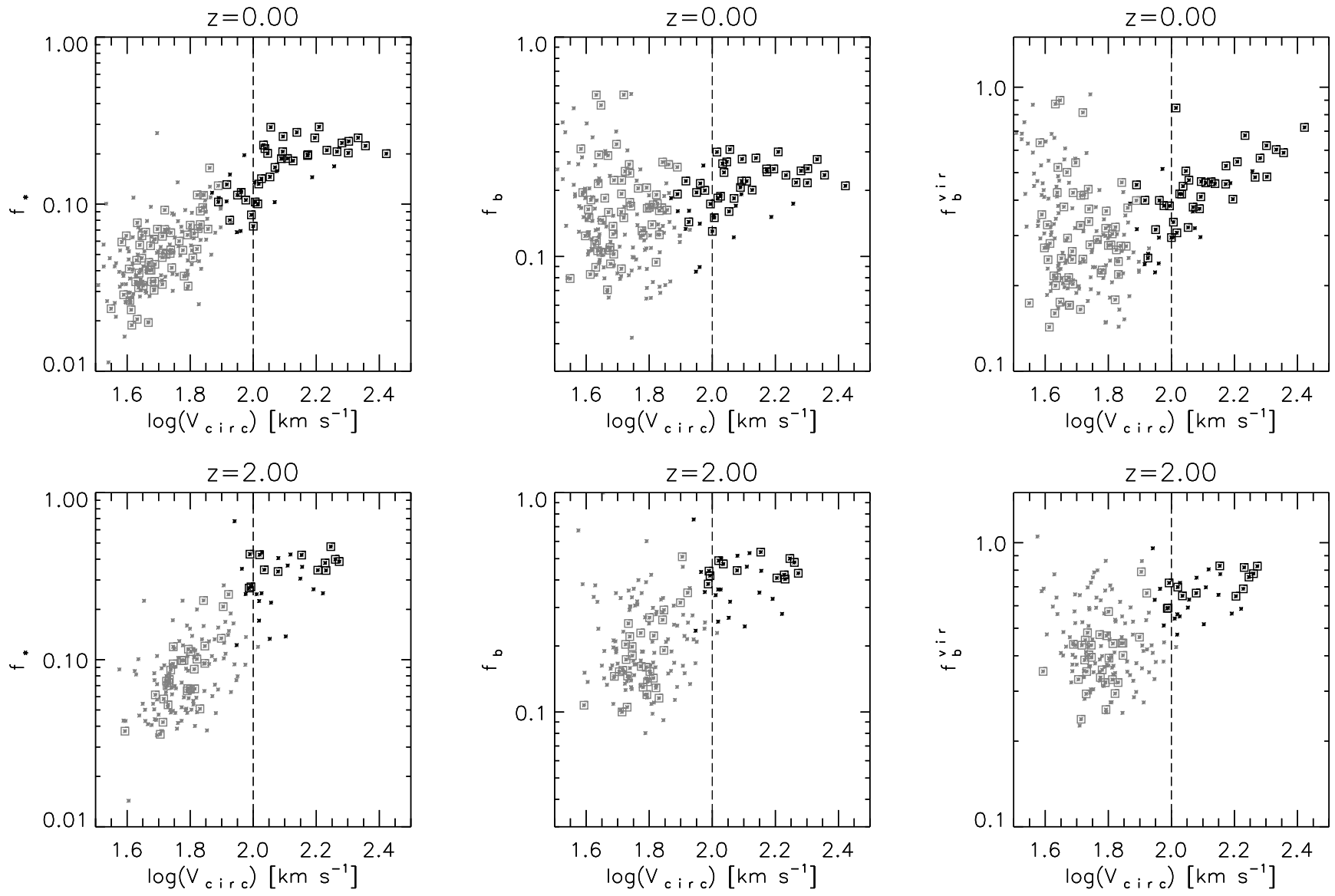

Fig. 7. Left and middle panels: Fraction of stellar $\left(f_{*}\right)$ and baryonic $\left(f_{\mathrm{b}}\right)$ mass residing in simulated galaxies relative to the expected baryonic mass within the virial radius for S230. Right panel: fraction of the total baryonic mass within the virial radius relative to the expected one $\left(f_{\mathrm{b}}^{\mathrm{vir}}\right)$ for S230. Results are shown at $z=0$ (upper panels) and $z=2$ (lower panels). For symbol codes see Fig. 2. The vertical axes have been plotted using logarithmic scale.

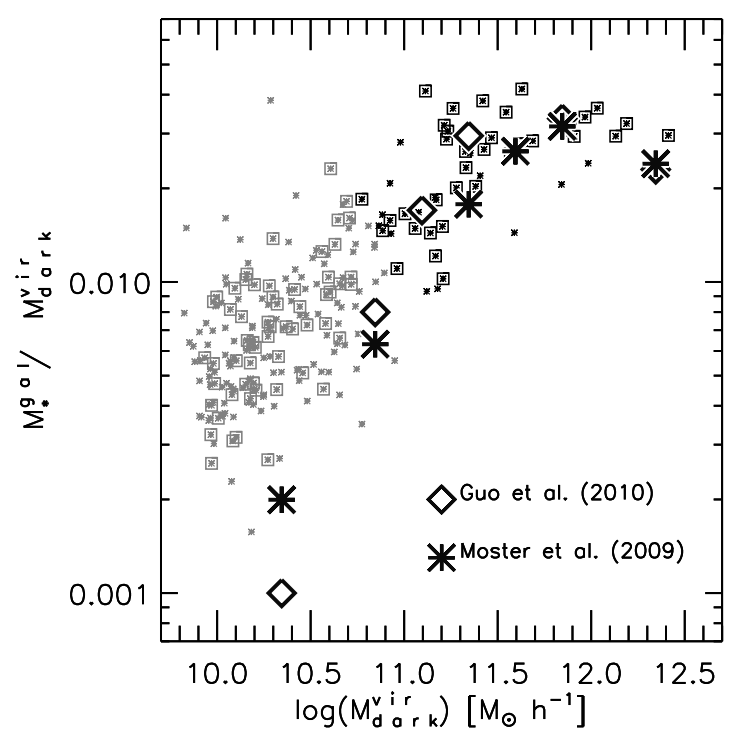

Fig. 8. Fraction of stellar mass residing in simulated galaxies at $z=0$ relative to the dark component within the virial radius. For symbol codes see Fig. 2. We show for comparison results from the semianalytical works by Guo et al. (2010) and Moster et al. (2009), as indicated in the figure. The vertical axis has been plotted using logarithmic scale.
Finally, in Fig. 8, we contrast our findings to the trends obtained from the semi-empirical models of Guo et al. (2010) and Moster et al. (2009) which are derived from the observed luminosity function. This figure shows the fraction of stellar mass $\left(F_{*}\right)$ contained within each simulated galaxy at $z=0$ relative to the dark component within the virial radius. We see that there is very good agreement between our results and previous works. However, our model still tends to overpredict the stellar mass fractions at the low-mass end of the relation.

The dynamical evolution of galaxies in our simulations is driven by the joint action of several physical processes, such as the star formation mechanism, gas infall, and galactic outflows. The star formation process is triggered mainly when the gas gets cold and dense ${ }^{2}$. As a consequence of star formation, SN energy is released, thereby heating up the surrounding cold and dense ISM, which when appropriated (see Sect. 2), is promoted to the hot phase. This generates a stronger decrease in the star formation activity in systems with shallower potential wells, because it is easier for the cold and dense gas in small systems to match the entropy of the hot and/or diffuse phase residing within their small dark matter haloes. But the cooling times $\left(t_{\text {cool }}\right)$ of the promoted particles are too short compared to their dynamical times

2 Recall that in this model the cold phase is defined as the gas component with temperature $T<T_{\mathrm{c}}$ where $T_{\mathrm{c}}=8 \times 10^{4} \mathrm{~K}$ and density $\rho>0.1 \rho_{\mathrm{c}}$ where $\rho_{\mathrm{c}}$ is $7 \times 10^{-26} \mathrm{~g} \mathrm{~cm}^{-3}$ (Sect. 2). Otherwise, the gas is classified as hot phase. 

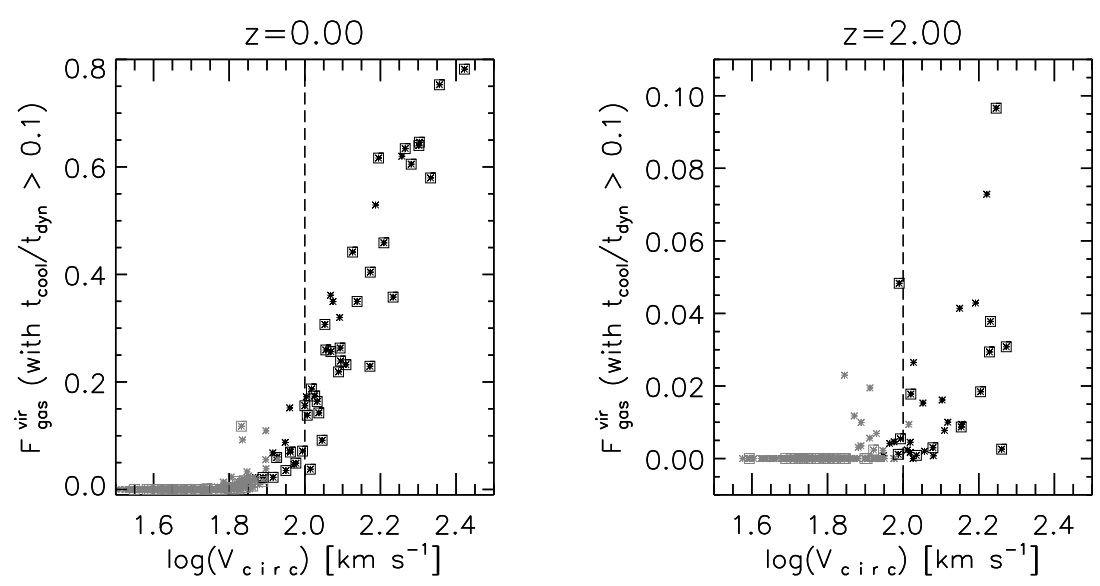

Fig. 9. Fraction of the gas component within $R_{\mathrm{vir}}$ that satisfies the condition $t_{\text {cool }} / t_{\mathrm{dyn}}>0.1$ in $\mathrm{S} 230$ at $z=0.0$ (left panel) and $z=2.0$ (right panel). For symbol codes see Fig. 2.

$\left(t_{\text {dyn }}\right)$ to allow this hot phase to be stable for a long time ${ }^{3}$. As a result, the gas that has just reached the hot phase due to SN heating might cool down again on short timescales, returning to the cold phase. Therefore, SN feedback leads to a self-regulated cycle of heating and cooling, exerting a strong influence on the regulation of the star formation process in low-mass galaxies. As the systems get larger, the hot phase is established at a higher temperature owing to the joint effect of the continuous pumping of SN energy into the hot environment and the increase in the virial temperature of the dark haloes hosting them. As the cooling times get longer than the dynamical time scales, the hot gas is able to remain in this phase. While the hot environment is able to build up as galaxies get more massive, galactic winds are more difficult to be triggered in these galaxies, since more SN energy would be required by the cold phase to match the entropy of its nearby hot environment and, therefore, to be promoted generating outflows. Meanwhile, the cold gas remains available for star formation. It is also true that, as the systems grow, their gas reservoir decreases, also decreasing their star formation activity and, consequently, the source of SN energy. Therefore, the action of $\mathrm{SN}$ feedback is much less important for regulating the transformation of the remaining gas into stars in these large systems.

To quantitatively check that these behaviours are taking place in our systems, we estimated the relations between the dynamical and the cooling times of the cold gas that has joined the hot phase by the action of SN feedback (i.e. promoted particles), during the evolution of our simulated volumes. The fraction of gas phase that is unavailable for efficient cooling within simulated galaxies $\left(F_{\text {gas }}^{\mathrm{vir}}\right.$ ) is defined as the gas mass within $R_{\text {vir }}$ that satisfies the condition $t_{\text {cool }} / t_{\text {dyn }}>0.1$ (Navarro \& White 1993). In Fig. 9, we plot $F_{\text {gas }}^{\mathrm{vir}}$ as a function of $V_{\text {circ }}$ in S230 at $z=0$ (left panel) and $z=2$ (right panel). As expected, $F_{\text {gas }}^{\mathrm{vir}}$ is an increasing function of $V_{\text {circ }}$. At $z=0$, systems with $V_{\text {circ }}<100 \mathrm{~km} \mathrm{~s}^{-1}$ have a significant percentage of their gas phase $(>90 \%)$ subject to efficient cooling. Only at $V_{\text {circ }}>100 \mathrm{~km} \mathrm{~s}^{-1}$ does $F_{\text {gas }}^{\text {vir }}$ start to increase, reaching 0.8 at $V_{\text {circ }} \sim 250 \mathrm{~km} \mathrm{~s}^{-1}$. The same trends are present at $z=2$, albeit with a larger dispersion at the highvelocity end. Interestingly, our model predicts that the transition from efficient to inefficient gas cooling occurs at around the characteristic velocity $V_{\mathrm{c}} \sim 100 \mathrm{~km} \mathrm{~s}^{-1}$ where the TFR bends. This

\footnotetext{
${ }^{3}$ In our model, the cooling and dynamical times are calculated following standard definitions as $t_{\text {cool }}=3 k T \mu \mathrm{m}_{\mathrm{p}} / 2 \Lambda \rho$ and $t_{\mathrm{dyn}}=\sqrt{3 \pi / 6 G \rho}$, respectively.
}

velocity also agrees with the theoretical expectations from Dekel \& Silk (1986), who concluded that, only for systems with $V_{\text {cir }}$ larger than $\sim 100 \mathrm{~km} \mathrm{~s}^{-1}$ are the cooling times of the diffuse and hot gas at least one order of magnitude longer than the dynamical times and, therefore, the gas could remain hot.

\section{Conclusions}

We have studied the sTFR and bTFR by performing hydrodynamical simulations in a cosmological scenario including the action of the combined multiphase and SN feedback model of Scannapieco et al. $(2005,2006)$. Our results suggest there is a change in the slope of the sTFR and bTFR produced by the action of SN feedback. At $z=0$, the simulated sTFR is very tightly defined and in general agreement with observations (e.g. McGaugh et al. 2000). We detect a systematical change in the slope of the sTFR from approximately $\sim 100 \mathrm{~km} \mathrm{~s}^{-1}$, at least since $z=3$, so that lower velocity systems tend to have lower stellar masses than those predicted by the linear regression of the fast rotators. The comparison with higher and lower resolution runs shows that this feature is not just a numerical artefact. Regarding the local bTFR, over the velocity range resolved by our simulations, it can be fitted by a single linear model. At higher redshifts, we detect a weak trend toward a change in the slope. Similar trends are found by using $V_{\max }$ as the kinematical estimator.

Our results suggest an evolution of $\approx 0.44$ dex from $z=3$ to $z=0$ for the sTFR, which is consistent with what is reported by Cresci et al. (2009) of $0.41 \pm 0.11$ dex from $z \approx 2.2$ to $z=0$. For the baryonic relation, our model predicts a slightly weaker evolution of $\approx 0.30$ dex from $z=3$ to $z=0$. In the wind-free run, both the sTFR and the bTFR show the same level of evolution $(\approx 0.55 \mathrm{dex})$, as expected since baryons are efficiently transformed into stars as soon as they collapse within the potential well of a galaxy.

The characteristic velocity of $\sim 100 \mathrm{~km} \mathrm{~s}^{-1}$ appears naturally as a consequence of the physically motivated multiphase and SN feedback treatment adopted in these simulations. This model is successful at establishing a self-regulated star formation process without using any global properties of the systems but resorting to the local estimations of the thermodynamical properties of the gas in the distinct components of the ISM. We checked that this result is robust against variations in the SN input parameters. Nevertheless, we acknowledge that there are several open 
questions that need to be solved in the future such as the level of change in the slope, which seems to be weaker in our simulations than in observations. In this regard, our findings suggest that the study of the sTFR and bTFR at different cosmic times could help constrain SN feedback models.

Acknowledgements. We thank the anonymous referee for his/her useful comments that largely helped to improve this paper. The authors are grateful to Cecilia Scannapieco for making the S320 simulation available and to Tomás Tecce for useful discussions. We acknowledge support from the PICT 32342 (2005) and PICT 245-Max Planck (2006) of ANCyT (Argentina). Simulations were run in Fenix and HOPE clusters at IAFE and Cecar cluster at University of Buenos Aires.

\section{References}

Amorín, R., Aguerri, J. A. L., Muñoz-Tuñón, C., \& Cairós, L. M. 2009, A\&A, 501,75

Avila-Reese, V., Firmani, C., \& Hernández, X. 1998, ApJ, 505, 37

Avila-Reese, V., Zavala, J., Firmani, C., \& Hernández-Toledo, H. M. 2008, AJ, 136, 1340

Bell, E. F., \& de Jong, R. S. 2001, ApJ, 550, 212

Conselice, C. J., Bundy, K., Ellis, R. S., et al. 2005, ApJ, 628, 160

Cresci, G., Hicks, E. K. S., Genzel, R., et al. 2009, ApJ, 697, 115

Croft, R. A. C., Di Matteo, T., Springel, V., \& Hernquist, L. 2009, MNRAS, 400, 43

De Rijcke, S., Zeilinger, W. W., Hau, G. K. T., Prugniel, P., \& Dejonghe, H. 2007, ApJ, 659, 1172

Dekel, A., \& Silk, J. 1986, ApJ, 303, 39

Dutton, A. A., \& van den Bosch, F. C. 2009, MNRAS, 396, 141

Flores, H., Hammer, F., Puech, M., Amram, P., \& Balkowski, C. 2006, A\&A, 455, 107

Governato, F., Willman, B., Mayer, L., et al. 2007, MNRAS, 374, 1479

Governato, F., Brook, C., Mayer, L., et al. 2010, Nature, 463, 203

Guo, Q., White, S., Li, C., \& Boylan-Kolchin, M. 2010, MNRAS, 404, 111

Gurovich, S., McGaugh, S. S., Freeman, K. C., et al. 2004, PASA, 21, 412

Gurovich, S., Freeman, K. C., Jerjen, H., Staveley-Smith, L., \& Puerani, I. 2010, AJ, 140, 663
Kang, X., Jing, Y. P., Mo, H. J., \& Borner, G. 2005, ApJ, 631, 21

Larson, R. B. 1974, MNRAS, 169, 229

Mac Low, M. M., \& Ferrara, A. 1999, ApJ, 513, 142

McGaugh, S. S. 2005, ApJ, 632, 859

McGaugh, S. S., Schombert, J. M., Bothun, G. D., \& de Blok, W. J. G. 2000, ApJ, 533, L99

McGaugh, S. S., Schombert, J. M., de Blok, W. J. G., \& Zagursky, M. J. 2010, ApJ, 708L, 14

Mo, H. J., Mao, S., \& White, S. D. M. 1998, MNRAS, 295, 319

Mosconi, M. B., Tissera, P. B., Lambas, D. G., \& Cora, S. A. 2001, MNRAS, 325,34

Moster, B. P., Somerville, R. S., Maulbetsch, C., et al. 2009, ApJ, 710, 903

Nagashima, M., Yahagi, H., Enoki, M., Yoshii, Y., \& Gouda, N. 2005, ApJ, 634, 26

Navarro, J. F., \& Steinmetz, M. 2000, ApJ, 538, 477

Navarro, J. F., \& White, S. D. M. 1993, MNRAS, 265, 271

Puech, M., Hammer, F., Flores, H., et al. 2010, A\&A, 510, 68

Raiteri, C. M., Villata, M., \& Navarro, J. F. 1996, A\&A, 315, 105

Scannapieco, C., Tissera, P. B., White, S. D. M., \& Springel, V. 2005, MNRAS, 364,552

Scannapieco, C., Tissera, P. B., White, S. D. M., \& Springel, V. 2006, MNRAS, 371,1125

Scannapieco, C., Tissera, P. B., White, S. D. M., \& Springel, V. 2008, MNRAS, 389,1137

Springel, V. 2005, MNRAS, 364, 1105

Springel, V., \& Hernquist, L. 2003, MNRAS, 339, 289

Springel, V., White, S. D. M., Tormen, G., \& Kauffmann, G. 2001, MNRAS, 328,726

Steinmetz, M., \& Navarro, J. F. 1999, ApJ, 513, 555

Stinson, G. S., Quinn, T., Dalcanton, J., Wadsley, J., \& Gogarten, S. 2007, A\&AS, 38, 766

Tassis, K., Kravtsov, A. V., \& Gnedin, N. Y. 2008, ApJ, 672, 888

Thielemann, F. K., Nomoto, K., \& Hashimoto, M. 1993, in Origin and Evolution of the Elements, ed. N. Prantzoz, et al. (Cambridge University Press), 297

Tissera, P. B., Lambas, D. G., \& Abadi, M. G. 1997, MNRAS, 286, 384

Tully, R. B., \& Fisher, J. R. 1977, A\&A, 54, 661

Verheijen, M. A. W. 2001, ApJ, 563, 694

White, S. D. M., \& Frenk, C. S. 1991, ApJ, 379, 52

Woosley, S. E., \& Weaver, T. A. 1995, ApJS, 101, 181 